\title{
Immunohistochemical Expression of MUC4 in Different Meningioma Subtypes in Comparison to Some Mesenchymal Non-Meningothelial Tumors
}

\author{
Kareman Mansour*(i), Dalal Anwar Elwi, Sara Elsayed Khalifa, Heba Abdelmonem Ibrahim \\ Department of Pathology, Faculty of Medicine, Cairo University, Giza, Egypt
}

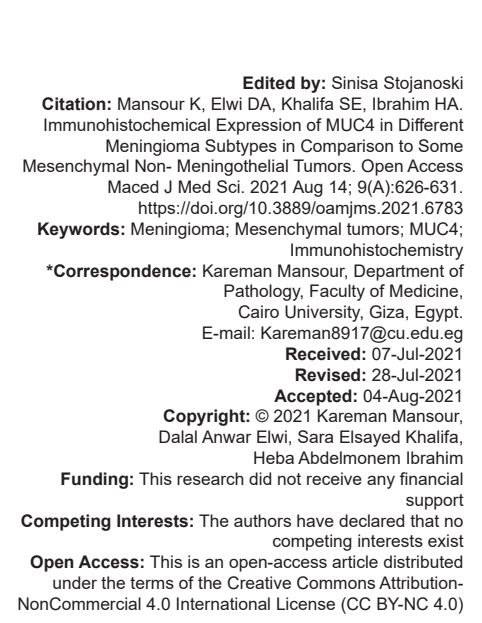

Abstract

BACKGROUND: Meningiomas are the most common primary tumors of the central nervous system worldwide. Routinely used immunohistochemical markers for diagnosis of confusing meningioma cases as epithelial membrane antigen lack specificity and sensitivity. MUC4 is glycosylated membrane-associated mucin expressed by normal epithelia and many cancers. However, it is recently noticed to be expressed in meningiomas.

AIM: Intensity of MUC4 expression is needed to be verified whether it is the same among different subtypes or not.

MATERIALS AND METHODS: Fifty cases of different intracranial meningioma subtypes and thirty cases of mesenchymal nonmeningothelial tumors were immunohistochemically stained with MUC4 antibody. The results of MUC4 expression intensity were associated with some clinical and pathological parameters.

RESULTS: Most studied meningioma cases (84\%) showed positive MUC4 expression. Meningothelial meningioma subtype showed characteristic pattern of diffuse and moderate to strong MUC4 staining. While fibroblastic meningioma showed mostly negative staining pattern and focal weak staining pattern if positive. A statistically significant relationship was detected between tumor subtype and intensity of MUC4 expression. On contrary, most included mesenchymal cases were MUC4 negative with statistical significance. Hence, the sensitivity of MUC4 as diagnostic marker for meningioma was $84 \%$, while the specificity was $93.3 \%$. Furthermore, meningioma histologic subtype showed significant relationship with age.

CONCLUSION: The current study results suggest that MUC4 could be used as meningioma diagnostic marker with some limitations. Moreover, meningioma should be included in the differential diagnosis of MUC4 positive tumors.

\section{Introduction}

Meningiomas constitute $36 \%$ of intracranial tumors and they are the most common primary tumors of the central nervous system (CNS) worldwide [1]. Benign meningiomas are the most common CNS tumors in the United States also, with increasing incidence in past decades but future levels will remain similar to current levels because of aging population [2]. They are also common in Egypt constituting 25.6\% of CNS tumors coming in the second place after glial tumors [3]. Meningiomas are divided into 15 histologic subtypes and three grades. Most of them are benign, the World Health Organization (WHO) Grade I, with a preferred prognosis. Grade II atypical meningiomas and grade III anaplastic meningiomas have less preferred outcomes [4], [5].

Diagnosis of meningioma is usually based on examination of Hematoxylin and Eosin ( $H$ \& $E$ ) sections; however, some problematic cases need further confirmation by immunohistochemistry. Despite epithelial membrane antigen (EMA) and progesterone receptor $(\mathrm{PgR})$ were used routinely for these cases, they proved to lack sensitivity and specificity, so a new sensitive and specific marker was needed [6].

In respect to their physiological and structural characteristics, mucins are further divided into two subfamilies; transmembrane mucins which include MUC4, and secreted mucins which form mucus layer acting as physical barrier protecting epithelial cells from stress-induced damage [7], [8].

Matsuyama et al., 2019 studied MUC4 expression in meningioma subtypes [9]. However, we noticed that MUC4 expression intensity was different among some subtypes. Moreover, they stated that specificity of MUC4 in meningioma was $100 \%$, but we encountered few positive mesenchymal nonmeningothelial cases. So, in the present study, we immunohistochemically studied MUC4 expression intensity in different meningioma subtypes and some mesenchymal nonmeningothelial tumors for comparison. 


\section{Materials and Methods}

This work included 50 cases of different intracranial meningioma subtypes and 30 cases of mesenchymal non meningothelial tumors, obtained through collection of archival paraffin blocks during the period from January 2017 till June 2019, from the Pathology department, Faculty of Medicine, Cairo University.

Data obtained from pathology sheet included age and sex of patient, site of lesion, and maximal diameter of the tumor. Two Serial sections of 4 microns thick were prepared from each paraffin block, one of them was mounted on glass slide and stained by H\&E for histological reevaluation according to the revised WHO classification 2016 [5], and the other one was mounted on charged slide for immunohistochemical staining.

Immunohistochemistry was performed using Dako Autostainer link 48 (Agilent, USA) according to the manufacturer's instructions and anti-MUC4 (Mouse Monoclonal, 1:100, Abbexa, USA, with Catalogue No.: abx173628). Antigen retrieval was done by immersing the slides in citrate buffer, $\mathrm{pH} 6$, for $20 \mathrm{~min}$ before the immunoreaction for MUC4. The expression of MUC4 in the tumor tissues was assessed using light microscope. Colonic mucosa and gastric carcinoma tissue were used as positive control for MUC4 protein expression and included in each run. As a negative control, the primary antibodies were replaced with saline.

The tumor was considered positive if $1 \%$ or more of tumor cells showed MUC4 cytoplasmic brown staining. The percentage of positive cells in each immunohistochemical slide was given a score from $1 \%$ up to $100 \%$ of tumor cells at $10 \%$ interval by three observers (K.M., S.K. and H.I.), and mean scores were reported. The expression of $50 \%$ or more of tumor cells was considered as diffuse staining [9]. The tumor cells which showed cytoplasmic staining were graded into four categories according to staining intensity:

(0) Negative: no detectable staining.

(1) Weak positivity: detectable, but not easily seen under a low-power objective (magnification; ×40).

(2) Moderate positivity: clearly positive, could be seen at low power objective.

(3) Strong positivity: heavy staining, intense, easily seen with a low-power objective.

For comparison, MUC4 expression was evaluated in thirty mesenchymal tumors arising from intracranial, spinal, and extracranial soft tissue locations.

The results of MUC4 immunostaining in meningioma tumor cells were tabulated to be associated with other clinicopathological parameters (age, sex, tumor size, and histopathologic subtype, WHO grade). Photos were taken by Leica EC4 camera using the Leica LAS EZ software. Data were coded and entered using the IBM SPSS Statistics version 26. Data were summarized using mean and standard deviation (SD) for quantitative variables and frequencies (number of cases) and relative frequencies (percentages) for categorical variables. Comparisons between groups were done using unpaired student test and one way Anova. For comparing categorical data, Chi-square $\left(\chi^{2}\right)$ test and Fisher Exact test were used. $p<0.05$ was considered as statistically significant.

\section{Results}

\section{Clinical data and histopathological findings}

The age of included meningioma patients ranged from 25 to 71 years with mean age $51 \pm 11.6$ $\mathrm{SD}$ years and median age 52 years. Female population (32 cases; 64\%) were more than male population (18 cases; $36 \%$ ) in studied patients. WHO Grade I was the most common grade encountered in studied meningioma cases $(80 \%)$ with transitional meningioma being the most encountered subtype (17 cases; $34 \%$ ) as demonstrated in Table 1. As regards to tumor size, it ranged from $2.5 \mathrm{~cm}$ up to $10 \mathrm{~cm}$ in maximum diameter with a mean diameter of $5.4 \mathrm{~cm} \pm 1.7 \mathrm{~cm}$ SD. Most meningioma cases (28 cases; $56 \%$ ) were $<5.4 \mathrm{~cm}$ in diameter.

Table 1: Distribution of clinicopathological variables in different meningioma subtypes

\begin{tabular}{|c|c|c|c|c|c|c|c|}
\hline & \multicolumn{2}{|c|}{ Age groups } & \multicolumn{2}{|l|}{ Gender } & \multicolumn{2}{|c|}{ Size groups } & \multirow[t]{2}{*}{ Total } \\
\hline & $<51$ year & $>51$ year & Male & Female & $<5.4 \mathrm{~cm}$ & $>5.4 \mathrm{~cm}$ & \\
\hline Meningothelial & $6(55 \%)$ & $5(45 \%)$ & $4(36 \%)$ & $7(63 \%)$ & $8(73 \%)$ & $3(27 \%)$ & 11 \\
\hline Fibroblastic & $5(100 \%)$ & $0(0 \%)$ & $1(20 \%)$ & $4(80 \%)$ & $2(40 \%)$ & $3(60 \%)$ & 5 \\
\hline Transitional & $6(35 \%)$ & $11(65 \%)$ & $6(35 \%)$ & $11(65 \%)$ & $9(53 \%)$ & $8(47 \%)$ & 17 \\
\hline Angiomatous & $0(0 \%)$ & $4(100 \%)$ & $2(50 \%)$ & $2(50 \%)$ & $2(50 \%)$ & $2(50 \%)$ & 4 \\
\hline Secretory & $1(100 \%)$ & $0(0 \%)$ & $0(0 \%)$ & $1(100 \%)$ & $1(100 \%)$ & $0(0 \%)$ & 1 \\
\hline Metaplastic & $1(100 \%)$ & $0(0 \%)$ & $0(0 \%)$ & $1(100 \%)$ & $1(100 \%)$ & $0(0 \%)$ & 1 \\
\hline Psammomatous & $0(0 \%)$ & $1(100 \%)$ & $0(0 \%)$ & $1(100 \%)$ & $1(100 \%)$ & $0(0 \%)$ & 1 \\
\hline Chordoid & $0(0 \%)$ & $1(100 \%)$ & $0(0 \%)$ & $1(100 \%)$ & $1(100 \%)$ & $0(0 \%)$ & 1 \\
\hline Clear cell & $1(100 \%)$ & $0(0 \%)$ & $1(100 \%)$ & $0(0 \%)$ & $0(0 \%)$ & $1(100 \%)$ & 1 \\
\hline Atypical & $2(29 \%)$ & $5(71 \%)$ & $3(43 \%)$ & $4(57 \%)$ & $3(43 \%)$ & $4(57 \%)$ & 7 \\
\hline Papillary & $0(0 \%)$ & $1(100 \%)$ & $1(100 \%)$ & $0(0 \%)$ & $0(0 \%)$ & $1(100 \%)$ & 1 \\
\hline Total & 22 & 28 & 18 & 32 & 28 & 22 & 50 \\
\hline$p$ value & $0.021^{*}$ & & 0.899 & & 0.756 & & \\
\hline
\end{tabular}

Regarding non-meningothelial mesenchymal tumors, Schwannoma was the most encountered of them (11 cases; $36.7 \%$, including five intracranial) followed by neurofibroma (8 cases; 26.7\%) and then Solitary fibrous tumor/hemangiopericytoma (SFT/HPC) (6 cases; 20\%) and hemangioblastoma (5 cases). The age of included patients ranged from 16 to 66 years with mean age $42 \pm 15.09$ SD years and median age 44 years. Female patient population $(53 \%)$ was slightly more than male patient population $(47 \%)$. The size ranged from $1 \mathrm{~cm}$ up to $10 \mathrm{~cm}$ in 
maximum diameter with mean size $4.5 \mathrm{~cm}$ in diameter. All included hemangioblastoma cases located in the cerebellum while most neurofibroma cases $(87.5 \%)$ located in extracranial soft tissue. Equal numbers of Schwannoma cases located in Cerebellopontine angle (CPA) and Spinal region. All included schwannoma cases, neurofibroma cases, hemangioblastoma cases were WHO Grade I. On the other hand, three cases of SFT/HPC cases were WHO Grade I, and only one case was WHO Grade III.

Histologic subtype showed significant relationship with age ( $p$ value; 0.02), all included fibroblastic cases were $<51$ years old, while all included angiomatous meningiomas were $>51$ years old.

\section{Immunohistochemical MUC4 expression}

Most studied meningioma cases (84\%) showed positive MUC4 expression. Meningothelial meningioma subtype showed the highest mean percentage of positive tumor cells (69\%), while transitional, angiomatous and atypical meningioma cases showing a mean $27 \%, 27.4 \%$ and $26.4 \%$ respectively. Fibroblastic meningioma showed the lowest mean percentage of positive tumor cells $(1 \%)$. Forty- four percent of cases showed diffuse staining ( $\geq 50 \%$ positive tumor cells) and 20 cases (40\%) have $1-50 \%$ positive tumor cells.

And as demonstrated in Table 2 and Figure 1, most meningothelial meningioma cases (91\%) showed strong and moderate staining intensity of MUC4 positive tumor cells while the only positive fibroblastic meningioma case showed weak staining intensity. On the other hand, weak intensity predominates in transitional meningioma cases (41\%) and atypical meningioma cases (57\%). A statistically significant

Table 2: Association between MUC4 expression intensity and other clinicopathological variants

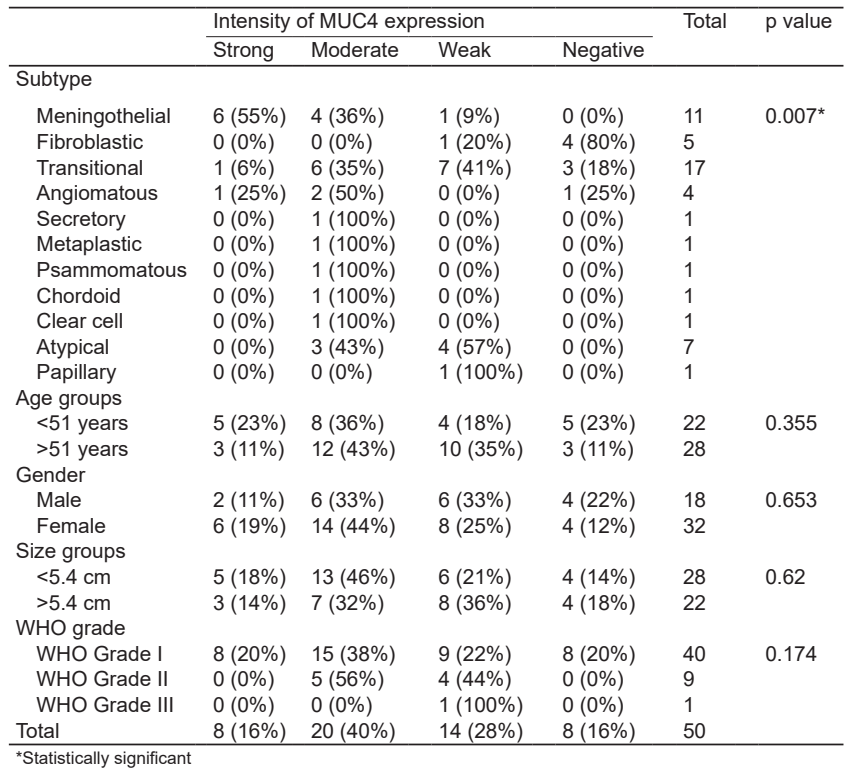

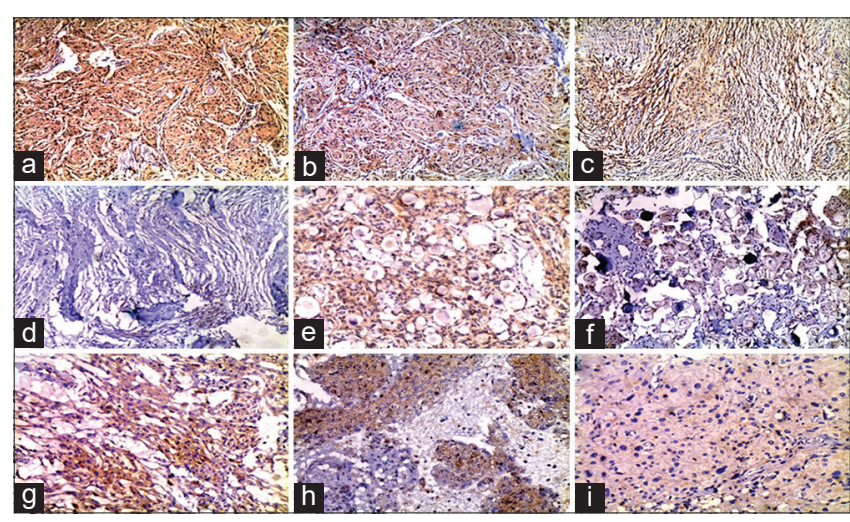

Figure 1: Immunohistochemical expression of MUC4 in meningioma subtypes; strong (a) and moderate (b) positive MUC4 expression in meningothelial meningioma ( $a, b, \times 100)$, moderate-intensity in transitional meningioma (c, $\times 100)$, weak in fibrous meningioma $(d, \times 200)$, moderate in secretory meningioma $(e, \times 100)$, psammomatous meningioma $(f, \times 100)$ and choroid meningioma $(g, \times 100)$, moderate (h) and weak (i) in atypical meningioma $(h, \times 200, i, \times 100)$

relationship was detected between intensity of MUC4 expression and tumor subtype with $p=0.007$. No statistically significant relationship could be detected between intensity of MUC4 expression and other parameters (age, gender, tumor size, and tumor grade)

On contrary, as shown in Figure 2, only two cases of included mesenchymal tumors $(6.7 \%)$ showed positive MUC4 expression. Only one case of schwannoma the WHO Grade I and one case of SFT/ HPT WHO Grade I showed $20 \%$ and $40 \%$ positive MUC4 tumor cells of weak and moderate-intensity, respectively.

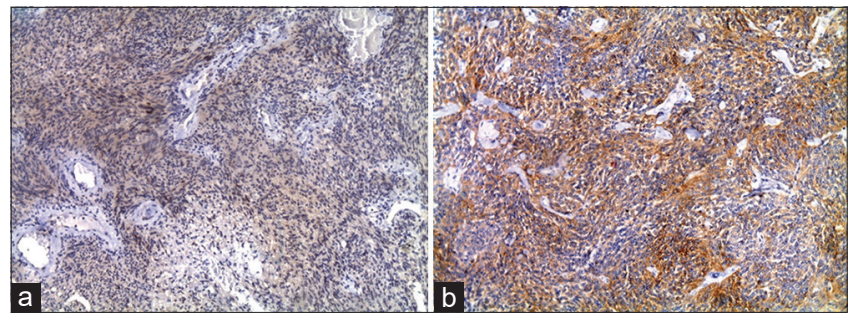

Figure 2: Weak immunohistochemical expression of MUC4 in a case of schwannoma $(a, \times 100)$, and moderate in a case of SFT/HPC WHO grade I $(b, \times 100)$

\section{Discussion}

Meningioma is the second most common CNS neoplasms in Egypt constituting 25.6\% of CNS tumors [3]. Although the routine immunohistochemical markers for diagnosis of confusing meningioma cases are EMA and PgR, they lack specificity and sensitivity in comparison to somatostatin receptor 2a for example [6]. In the current study, MUC4 immunoreactivity, intensity of staining in positive cases, and percentage of positive tumor cells were examined in tumor cells 
of fifty meningioma cases and thirty mesenchymal nonmeningothelial tumor cases to assess MUC4 utility as a diagnostic marker for meningioma.

Theage ofincluded meningioma patients ranged from 25 to 71 years with mean age of 51 and median age of 52 years. Some studies reported similar results and others reported higher figures [9], [10], [11], [12]. Also, Central Brain Tumor Registry of the United States (CBTRUS) statistical reports reported that median age at diagnosis was 66 years old [13]. Relatively, presence of younger mean age in our study may be due to difference in sample size. Females represented 64\% of the present study population. This was more or less similar to that found in literature and CBTRUS statistical reports [3], [9], [10], [11], [12], [13].

Tumor size ranged from $2.5 \mathrm{~cm}$ up to $10 \mathrm{~cm}$ in maximum diameter with mean size of $5.4 \mathrm{~cm}$ in diameter. Matsuyama et al. in 2019 found that the sizes of the tumor more widely ranged from 0.9 to $12 \mathrm{~cm}$ with mean size $3.6 \mathrm{~cm}$ [9]. Higher mean size in this study partially suggested by the limited availability of diagnostic imaging modalities in secondary health care centers in Egypt delaying diagnosis.

The WHO Grade I was the most common grade encountered in our studied meningioma cases $(80 \%)$, similar to other studies but with varying proportions [6], [9], [10], [11], [12]. Transitional meningioma was the most encountered subtype $(34 \%)$ in our work followed by meningothelial meningioma (22\%). Similar to our results, Ding et al. in 2014 noted that $32 \%$ of their cases were transitional meningioma [11]. However, the meningothelial meningioma subtype was the most encountered subtype in other studies [3], [9], [10]. Surprisingly, Kato et al. in 2014 reported that $35.5 \%$ of their cases were atypical meningioma [12]. These different figures can be explained partially by different sample sizes. In addition, most of these studies were conducted before the last WHO classification in 2016 which introduce brain invasion alone as a criterion for diagnosis of atypical meningioma (WHO grade II).

To the best of our knowledge, no enough literature is available to suggest a link between the histological subtypes of meningiomas and patient's age at diagnosis. In our study, a significant relationship with age ( $p$ value; 0.02) was reported with all included fibroblastic cases and most meningothelial cases were $<51$ years old, while, all included angiomatous meningiomas, most of atypical meningioma cases were $>51$ years old. however, further research with larger sample size is needed to establish this relationship.

As regards to included mesenchymal tumors in our study, all included hemangioblastoma cases located in the cerebellum while most neurofibroma cases $(87.5 \%)$ were located in extracranial soft tissue. Equal numbers of schwannoma cases located in CPA and spinal region. This was inconsistent with what reported in literature [5], [14].

To the best of our knowledge, apart from Matsuyama et al. study in 2019, no other works studied MUC4 expression in meningioma and other mesenchymal tumors. In our study, MUC4 was expressed in $84 \%$ of meningioma cases, although with different proportion of positive tumor cells among different subtypes. This is somewhat similar to what was reported by Matsuyama et al. in 2019 who found $92.9 \%$ of their studied meningioma cases were MUC4 positive [9]. Moreover, in our study, meningothelial meningioma subtype showed the highest mean percentage of positive tumor cells (69\%), while fibroblastic meningioma showed the lowest mean percentage of positive tumor cells (1\%). Similarly, Matsuyama et al. in 2019 reported that mean percentage of positive tumor cells in meningothelial and fibrous meningioma cases were $64.3 \%$ and $3.1 \%$, respectively. However, they reported that the mean percentage of positive cells in angiomatous meningioma were $88 \%$ [9]. This was contrary to our results that showed that the mean percentage of positive tumor cells in angiomatous meningioma cases was $27.5 \%$. A possible explanation for this could be the small number of angiomatous meningioma cases in our sample.

In our study, most transitional meningioma cases (82\%) showed positive MUC4 expression while most included fibroblastic meningioma (80\%) showed negative MUC4 expression. These results were in partial agreement with Matsuyama et al. in 2019 but most of their studied fibrous meningioma cases (70\%) were MUC4 positive (albeit focally) in contrary to our results [9]. This is partially due to limited number of fibrous meningioma cases in our sample.

Regarding staining intensity in the current study, meningothelial meningioma showed a characteristic pattern of diffuse and moderate to strong MUC4 staining. On the other hand, fibrous meningiomas showed mostly negative staining pattern, and focal weak staining pattern if positive, so not surprisingly that transitional meningioma cases showed intermediate pattern of staining. Unfortunately, there was no data in literature about the intensity of MUC4 staining in meningioma cases to compare our results with.

MUC4 is expressed normally by tissue epithelia and different carcinomas, so it is mainly an epithelial marker [15]. Hence, it is suspected to be expressed in this diffuse and strong pattern in meningothelial meningioma which exhibits epithelioid cell morphology with syncytial-like appearance in the contrary to fibrous meningioma which showed spindled cell morphology. This expression might represent genetic or epigenetic changes needing further genetic studies to exclude or prove that. 
Only two cases of studied mesenchymal tumors $(6.7 \%)$ out of 30 cases showed positive MUC4 expression, so, most studied meningioma cases were MUC4 positive, while most included mesenchymal cases were MUC4 negative with statistical significance ( $p$ value; 0.00001). These positive two mesenchymal cases decrease specificity of MUC4 as a meningioma diagnostic marker to $93.3 \%$. This was slightly different from Matsuyama et al. study in 2019 who reported that all their included mesenchymal tumors were MUC4 negative with $100 \%$ specificity of MUC4 as a meningioma diagnostic marker [9]. This difference needed to be verified with larger sample size.

On the other hand, gene expression profiling and immunohistochemical analyses revealed MUC4 expression in low grade fibromyxoid sarcoma (LGFMS) and sclerosing epithelioid fibrosarcoma (SEF) [16], [17]. Taking this into consideration allowed not to diagnose MUC4 spindle positive tumor as LGFMS or SEF, also not to diagnose any MUC4 positive epithelioid tumor as metastatic carcinoma [9].

In contrary to MUC4 expression in cancers, MUC4 in the current study less obviously expressed in high-grade meningiomas in comparison to $\mathrm{WHO}$ grade I meningioma cases as MUC4 expressed weakly in most atypical meningioma cases $(57 \%)$ and in the included papillary meningioma case with positivity limited to $<50 \%$ of tumor cells. These findings needed to be explained by further molecular studies.

\section{Conclusion}

The current study results suggest that MUC4 could be used as meningioma diagnostic marker with some limitations. Moreover, meningioma should be included in differential diagnosis of MUC4 positive tumors. However, the study has some limitations due to small number of cases and limited number of some rare subtypes. So, studies for MUC4 expression with larger sample size and involvement of more uncommon subtypes are needed to verify these results. Molecular studies also needed to clarify if MUC4 expression in meningioma represent aberrant genetic events or not.

\section{References}

1. Louis DN, Perry A, Reifenberger G, Von Deimling A, Figarella-Branger D, Cavenee WK, et al. The 2016 World Health Organization classification of tumors of the central nervous system: A summary. Acta Neuropathol. 2016;131(6):803-20. https://doi.org/10.1007/s00401-016-1545-1

PMid:27157931
2. Bhala S, Stewart DR, Kennerley V, Petkov VI, Rosenberg PS, Best AF. Incidence of benign meningiomas in the United States: Current and future trends. JNCI Cancer Spectrum. 2021;5(3):35. https://doi.org/10.1093/jncics/pkab035

3. Zalata KR, El-Tantawy DA, Abdel-Aziz A, Ibraheim AW, Halaka $\mathrm{AH}$, Gawish $\mathrm{HH}$, et al. Frequency of central nervous system tumors in delta region, Egypt. Indian J Pathol Microbiol. 2011;54(2):299. https://doi.org/10.4103/0377-4929.81607 PMid:21623078

4. Choy W, Ampie L, Lamano JB, Kesavabhotla K, Mao Q, Parsa AT, et al. Predictors of recurrence in the management of chordoid meningioma. J Neurooncol. 2016;126(1):107-16. https://doi.org/10.1007/s11060-015-1940-9 PMid:26409888

5. Louis DN, Ohgaki H, Wiestler OD, Cavenee WK. WHO Classification of Tumors of the Central Nervous System Revised. $4^{\text {th }}$ ed. Lyon, France: International Agency for Research on Cancer; 2016. p. 1-355.

6. Menke JR, Raleigh DR, Gown AM, Thomas S, Perry A, Tihan T. Somatostatin receptor $2 a$ is a more sensitive diagnostic marker of meningioma than epithelial membrane antigen. Acta Neuropathol. 2015;130(3):441-3. https://doi.org/10.1007/ s00401-015-1459-3

PMid:26195322

7. Hollingsworth MA, Swanson BJ. Mucins in cancer: Protection and control of the cell surface. Nat Rev Cancer. 2004;4(1):4560. https://doi.org/10.1038/nrc1251

PMid:14681689

8. Kaur S, Kumar S, Momi N, Sasson AR, Batra SK. Mucins in pancreatic cancer and its microenvironment. Nat Rev Gastroenterol Hepatol. 2013;10(10):607. https://doi. org/10.1038/nrgastro.2013.120

PMid:23856888

9. Matsuyama A, Jotatsu M, Uchihashi K, Tsuda Y, Shiba E, Haratake J, et al. MUC 4 expression in meningiomas: Underrecognized immunophenotype particularly in meningothelial and angiomatous subtypes. Histopathology. 2019;74(2):276-83 https://doi.org/10.1111/his.13730 PMid:30112770

10. de Oliveira Silva CB, Ongaratti BR, Trott G, Haag T, Ferreira NP, Leães CG, et al. Expression of somatostatin receptors (SSTR1SSTR5) in meningiomas and its clinicopathological significance. Int J Clin Exp Pathol. 2015;8(10):13185.

PMid:26722517

11. Ding Y, Qiu L, Xu Q, Song L, Yang S, Yang T. Relationships between tumor microenvironment and clinicopathological parameters in meningioma. Int $\mathrm{J}$ Clin Exp Pathol. 2014;7(10):6973.

PMid:25400783

12. Kato $\mathrm{Y}$, Nishihara $\mathrm{H}$, Mohri $\mathrm{H}$, Kanno $\mathrm{H}$, Kobayashi $\mathrm{H}$, Kimura $\mathrm{T}$, et al. Clinicopathological evaluation of cyclooxygenase-2 expression in meningioma: Immunohistochemical analysis of 76 cases of low and high-grade meningioma. Brain Tumor Pathol. 2014;31(1):23-30. https://doi.org/10.1007/s10014-012-0127-8. PMid:23250387

13. Ostrom QT, Gittleman H, Truitt G, Boscia A, Kruchko C Barnholtz-Sloan JS. CBTRUS statistical report: Primary brain and other central nervous system tumors diagnosed in the United States in 2011-2015. Neurooncology. 2018;20(4):1-86. https://doi.org/10.1093/neuonc/noy131 PMid:30445539

14. Pierscianek D, Wolf $S$, Keyvani K, El Hindy N, Stein KP, Sandalcioglu IE, et al. Study of angiogenic signaling pathways in hemangioblastoma. Neuropathology. 2017;37(1):3-11. https:// doi.org/10.1111/neup. 12316 


\section{PMid:27388534}

15. Chaturvedi P, Singh AP, Batra SK. Structure, evolution, and biology of the MUC4 mucin. FASEB J. 2008;22(4):966-81. https://doi.org/10.1096/fj.07-9673rev

PMid: 18024835

16. Doyle LA, Möller E, Dal Cin P, Fletcher CD, Mertens F, Hornick JL. MUC4 is a highly sensitive and specific marker for low-grade fibromyxoid sarcoma. Am J Surg Pathol. 2011;35(5):733-41. https://doi.org/10.1097/pas.0b013e318210c268

PMid:21415703

17. Doyle LA, Wang WL, Dal Cin P, Lopez-Terrada D, Mertens F, Lazar AJ, et al. MUC4 is a sensitive and extremely useful marker for sclerosing epithelioid fibrosarcoma: Association with FUS gene rearrangement. Am J Surg Pathol. 2012;36(10):1444-51. https://doi.org/10.1097/pas.0b013e3182562bf8

PMid:22982887 